\title{
Traveling Wave Solutions of Some Coupled Nonlinear Evolution Equations
}

\author{
Kamruzzaman Khan ${ }^{1}$ and M. Ali Akbar ${ }^{2}$ \\ ${ }^{1}$ Department of Mathematics, Pabna University of Science and Technology, Pabna 6600, Bangladesh \\ ${ }^{2}$ Department of Applied Mathematics, University of Rajshahi, Rajshahi 6205, Bangladesh
}

Correspondence should be addressed to Kamruzzaman Khan; k.khanru@gmail.com

Received 2 April 2013; Accepted 28 April 2013

Academic Editors: A. M. Gavrilik and G. F. Torres del Castillo

Copyright (C) 2013 K. Khan and M. A. Akbar. This is an open access article distributed under the Creative Commons Attribution License, which permits unrestricted use, distribution, and reproduction in any medium, provided the original work is properly cited.

The modified simple equation (MSE) method is executed to find the traveling wave solutions for the coupled Konno-Oono equations and the variant Boussinesq equations. The efficiency of this method for finding exact solutions and traveling wave solutions has been demonstrated. It has been shown that the proposed method is direct, effective, and can be used for many other nonlinear evolution equations (NLEEs) in mathematical physics. Moreover, this procedure reduces the large volume of calculations.

\section{Introduction}

Nowadays NLEEs have been the subject of all-embracing studies in various branches of nonlinear sciences. A special class of analytical solutions named traveling wave solutions for NLEEs has a lot of importance, because most of the phenomena that arise in mathematical physics and engineering fields can be described by NLEEs. NLEEs are frequently used to describe many problems of protein chemistry, chemically reactive materials, in ecology most population models, in physics the heat flow and the wave propagation phenomena, quantum mechanics, fluid mechanics, plasma physics, propagation of shallow water waves, optical fibers, biology, solid state physics, chemical kinematics, geochemistry, meteorology, electricity, and so forth. Therefore, investigation, traveling wave solutions is becoming more and more attractive in nonlinear sciences day by day. However, not all equations posed of these models are solvable. As a result, many new techniques have been successfully developed by diverse groups of mathematicians and physicists, such as the modified simple equation method [1-4], the extended tanh method [5, 6], the Exp-function method [7-11], the Adomian decomposition method [12], the F-expansion method [13], the auxiliary equation method [14], the Jacobi elliptic function method [15], modified Exp-function method [16], the $\left(G^{\prime} / G\right)$-expansion method [17-26], Weierstrass elliptic function method [27], the homotopy perturbation method [28-30], the homogeneous balance method [31, 32], the Hirota's bilinear transformation method $[33,34]$, the tanhfunction method $[35,36]$ and so on.

The objective of this paper is to apply the MSE method to construct the exact and traveling wave solutions for nonlinear evolution equations in mathematical physics via coupled Konno-Oono equations and variant Boussinesq equations.

The paper is prepared as follows. In Section 2, the MSE method is discussed. In Section 3, we apply this method to the nonlinear evolution equations pointed out above, in Section 4, physical explanations, and in Section 5 conclusions are given.

\section{The MSE Method}

In this section, we describe the MSE method for finding traveling wave solutions of nonlinear evolution equations. Suppose that a nonlinear equation, say in two independent variables $x$ and $t$, is given by

$$
\mathscr{R}\left(u, u_{t}, u_{x}, u_{t t}, u_{x x}, u_{x t}, \ldots\right)=0,
$$

where $u(\xi)=u(x, t)$ is an unknown function, $\mathscr{R}$ is a polynomial of $u(x, t)$ and its partial derivatives in which the highest order derivatives and nonlinear terms are involved. In the following, we give the main steps of this method [1-4]. 
Step 1. Combining the independent variables $x$ and $t$ into one variable $\xi=x \pm \omega t$, we suppose that

$$
u(\xi)=u(x, t), \quad \xi=x \pm \omega t .
$$

The traveling wave transformation equation (2) permits us to reduce (1) to the following ODE:

$$
\mathscr{R}\left(u, u^{\prime}, u^{\prime \prime}, \ldots\right)=0,
$$

where $\mathscr{R}$ is a polynomial in $u(\xi)$ and its derivatives, while $u^{\prime}(\xi)=d u / d \xi, u^{\prime \prime}(\xi)=d^{2} u / d \xi^{2}$, and so on.

Step 2. We suppose that (3) has the formal solution

$$
u(\xi)=C_{0}+\sum_{k=1}^{n} C_{k}\left(\frac{\phi^{\prime}(\xi)}{\phi(\xi)}\right)^{k}
$$

where $C_{k}$ are constants to be determined, such that $C_{n} \neq 0$, and $\phi(\xi)$ is an unknown function to be determined later.

Step 3. The positive integer $n$ can be determined by considering the homogeneous balance between the highest order derivatives and the nonlinear terms appearing in (1) or (3). Moreover precisely, we define the degree of $u(\xi)$ as $D(u(\xi))=$ $n$ which gives rise to the degree of other expression as follows:

$$
\begin{gathered}
D\left(\frac{d^{q} u}{d \xi^{q}}\right)=n+q, \\
D\left(u^{p}\left(\frac{d^{q} u}{d \xi^{q}}\right)^{s}\right)=n p+s(n+q) .
\end{gathered}
$$

Therefore, we can find the value of $n$ in (4), using (5).

Step 4. We substitute (4) into (3), and then we account the function $\phi(\xi)$. As a result of this substitution, we get a polynomial of $\left(\phi^{\prime}(\xi) / \phi(\xi)\right)$ and its derivatives. In this polynomial, we equate the coefficients of same power of $\phi^{-i}(\xi)$ to zero, where $i \geq 0$. This procedure yields a system of equations which can be solved to find $\alpha_{k}, \phi(\xi)$ and $\phi^{\prime}(\xi)$. Then the substitution of the values of $\alpha_{k}, \phi(\xi)$ and $\phi^{\prime}(\xi)$ into (4) completes the determination of exact solutions of (1).

\section{Applications}

3.1. The New Coupled Konno-Oono Equations. Now we will bring to bear the MSE method to find exact solutions, and then the solitary wave solutions of coupled Konno-Oono equations in the form [37],

$$
u_{x t}-2 u v=0, \quad v_{t}+2 u u_{x}=0 .
$$

Now let us suppose that the traveling wave transformation equation be

$$
u(\xi)=u(x, t), \quad v(\xi)=v(x, t), \quad \xi=x-\omega t .
$$

Equation (7) reduces (6) into the following ODEs:

$$
\begin{aligned}
& -\omega u^{\prime \prime}-2 u v=0, \\
& -\omega v^{\prime}+2 u u^{\prime}=0 .
\end{aligned}
$$

By integrating (9) with respect to $\xi$, we obtain

$$
v=\frac{1}{\omega}\left(u^{2}+d\right)
$$

where $d$ is a constant of integration.

Substituting (10) into (8), we get

$$
\omega^{2} u^{\prime \prime}+2 u d+2 u^{3}=0 .
$$

Balancing the highest order derivative $u^{\prime \prime}$ and nonlinear term $u^{3}$ from (11), we obtain $3 n=n+2$, which gives $n=1$.

Now for $n=1$, using (4) we can write

$$
u(\xi)=C_{0}+C_{1}\left(\frac{\phi^{\prime}(\xi)}{\phi(\xi)}\right),
$$

where $C_{0}$ and $C_{1}$ are constants to be determined such that $C_{1} \neq 0$, while $\phi(\xi)$ is an unknown function to be determined. It is trouble free to find that

$$
\begin{gathered}
u^{\prime}=C_{1}\left(\frac{\phi^{\prime \prime}}{\phi}-\left(\frac{\phi^{\prime}}{\phi}\right)^{2}\right), \\
u^{\prime \prime}=C_{1}\left(\frac{\phi^{\prime \prime \prime}}{\phi}\right)-3 C_{1}\left(\frac{\phi^{\prime \prime} \phi^{\prime}}{\phi^{2}}\right)+2 C_{1}\left(\frac{\phi^{\prime}}{\phi}\right)^{3}, \\
u^{3}=C_{1}^{3}\left(\frac{\phi^{\prime}}{\phi}\right)^{3}+3 C_{1}^{2} C_{0}\left(\frac{\phi^{\prime}}{\phi}\right)^{2}+3 C_{1} C_{0}^{2}\left(\frac{\phi^{\prime}}{\phi}\right)+C_{0}^{3} .
\end{gathered}
$$

Now substituting the values of $u, u^{3}, u^{\prime \prime}$ into (11) and then equating the coefficients of $\phi^{0}, \phi^{-1}, \phi^{-2}, \phi^{-3}$ to zero, we, respectively, obtain

$$
\begin{gathered}
2 C_{0}^{3}+2 A C_{0}=0, \\
\omega^{2} C_{1} \phi^{\prime \prime \prime}+6 C_{0}^{2} C_{1} \phi^{\prime}+2 A C_{1} \phi^{\prime}=0 \\
-3 \omega^{2} C_{1} \phi^{\prime \prime} \phi^{\prime}+6 C_{0} C_{1}^{2}\left(\phi^{\prime}\right)^{2}=0, \\
2 \omega^{2} C_{1}\left(\phi^{\prime}\right)^{3}+2 C_{1}^{3}\left(\phi^{\prime}\right)^{3}=0 .
\end{gathered}
$$

Solving (14), we get

$$
C_{0}=0, \pm \sqrt{-d}
$$

Solving (17), we get

$$
C_{1}= \pm I \omega, \quad C_{1} \neq 0, \quad \text { where } I^{2}=-1 .
$$

Solving (15) and (16) we get,

$$
\phi^{\prime}(\xi)=M A \exp (L M \xi)
$$

Integrating (20) with respect to $\xi$, we obtain

$$
\phi(\xi)=\frac{1}{L}(L B-A \exp (L M \xi))
$$


where $L=\left(6 C_{0}^{2}+2 d\right) / \omega^{2}, M=\omega^{2} / 2 C_{1} C_{0}$, and $A, B$ are constants of integration.

Substituting the values of $\phi$ and $\phi^{\prime}$ into (12), we obtain the following exact solution:

$$
u(x, t)=C_{0}+C_{1} \frac{L M A \exp (-L M(x-\omega t))}{L B-A \exp (-L M(x-\omega t))} .
$$

Case 1. When $C_{0}=0,(22)$ yields trivial solution. So this case is discarded.

Case 2. When $C_{0}= \pm \sqrt{-d}$ and $C_{1}= \pm I \omega$, substituting the values of $C_{0}, C_{1}, L, M$ into (22), we obtain

$$
\begin{aligned}
& u(x, t)= \pm I \sqrt{d} \\
& \times\left(1+\left(2 A \cosh \left(\frac{\sqrt{d}}{\omega}(x-\omega t)\right)\right.\right. \\
&\left.\quad-\sinh \left(\frac{\sqrt{d}}{\omega}(x-\omega t)\right)\right) \\
& \quad(L B-A) \cosh \left(\frac{\sqrt{d}}{\omega}(x-\omega t)\right) \\
&\left.\left.\quad+(L B+A) \sinh \left(\frac{\sqrt{d}}{\omega}(x-\omega t)\right)\right)^{-1}\right) .
\end{aligned}
$$

We can freely choose the constants $A$ and $B$. Therefore, setting $A=L B$, (23) reduces to

$$
u_{1,2}(x, t)= \pm I \sqrt{d} \operatorname{coth}\left(\frac{\sqrt{d}}{\omega}(x-\omega t)\right), \quad \text { for } d>0 \text {. }
$$

Again, if we set $A=-L B,(23)$ reduces to

$$
u_{3,4}(x, t)= \pm I \sqrt{d} \tanh \left(\frac{\sqrt{d}}{\omega}(x-\omega t)\right), \quad \text { for } d>0 \text {. }
$$

Substituting (24) and (25) into (10), we get

$$
\begin{gathered}
v_{1}(x, t)=-\frac{d}{\omega} \operatorname{cosech}^{2}\left(\frac{\sqrt{d}}{\omega}(x-\omega t)\right), \quad \text { for } d>0, \\
v_{2}(x, t)=\frac{d}{\omega} \operatorname{sech}^{2}\left(\frac{\sqrt{d}}{\omega}(x-\omega t)\right), \quad \text { for } d>0,
\end{gathered}
$$

respectively.
If $d<0$, using hyperbolic function identities, from (24)(27), we get the following periodic travelling wave solutions:

$$
\begin{gathered}
u_{5,6}(x, t)= \pm \sqrt{d} \cot \left(\frac{\sqrt{-d}}{\omega}(x-\omega t)\right), \\
u_{7,8}(x, t)= \pm \sqrt{d} \tan \left(\frac{\sqrt{-d}}{\omega}(x-\omega t)\right), \\
v_{3}(x, t)=\frac{d}{\omega} \operatorname{cosec}^{2}\left(\frac{\sqrt{-d}}{\omega}(x-\omega t)\right), \\
v_{4}(x, t)=\frac{d}{\omega} \sec ^{2}\left(\frac{\sqrt{-d}}{\omega}(x-\omega t)\right) .
\end{gathered}
$$

3.2. The Variant Boussinesq Equations. In this section, we will apply the modified simple equation method to find the exact solutions and then the solitary wave solutions of the variant Boussinesq equation [24] in the form

$$
u_{t}+H_{x}+u u_{x}=0, \quad H_{t}+(u H)_{x}+u_{x x x}=0
$$

The traveling wave transformation is

$$
u(\xi)=u(x, t), \quad H(\xi)=H(x, t), \quad \xi=x-\omega t .
$$

Using traveling wave equation (33), (32) reduces into the following ODEs:

$$
\begin{gathered}
-\omega u^{\prime}+H^{\prime}+u u^{\prime}=0 . \\
-\omega H^{\prime}+(u H)^{\prime}+u^{\prime \prime \prime}=0 .
\end{gathered}
$$

Integrating (34) with respect to $\xi$, choosing constant of integration as zero, we obtain the following ODEs:

$$
\begin{gathered}
-\omega u+H+\frac{1}{2} u^{2}=0, \\
-\omega H+u H+\frac{1}{3} u^{\prime \prime}=0 .
\end{gathered}
$$

From (35), we get

$$
H=\omega u-\frac{1}{2} u^{2}
$$

Substituting (37) into (36) yields

$$
u^{\prime \prime}-\omega^{2} u+\frac{3}{2} \omega u^{2}-\frac{1}{2} u^{3}=0
$$

Now balancing the highest order derivative $u^{\prime \prime}$ and nonlinear term $u^{3}$, we get $n=1$.

Now for $n=1, u(\xi)=C_{0}+\sum_{k=1}^{n} C_{k}\left(\phi^{\prime}(\xi) / \phi(\xi)\right)^{k}$ becomes

$$
u(\xi)=C_{0}+C_{1}\left(\frac{\phi^{\prime}(\xi)}{\phi(\xi)}\right)
$$


where $C_{0}$ and $C_{1}$ are constants to be determined such that $C_{1} \neq 0$, while $\phi(\xi)$ is an unknown function to be determined. It is easy to see that

$$
\begin{gathered}
u^{\prime}=C_{1}\left(\frac{\phi^{\prime \prime}}{\phi}-\left(\frac{\phi^{\prime}}{\phi}\right)^{2}\right), \\
u^{\prime \prime}=C_{1}\left(\frac{\phi^{\prime \prime \prime}}{\phi}\right)-3 C_{1}\left(\frac{\phi^{\prime \prime} \phi^{\prime}}{\phi^{2}}\right)+2 C_{1}\left(\frac{\phi^{\prime}}{\phi}\right)^{3}, \\
u^{2}=C_{0}^{2}+2 C_{0} C_{1}\left(\frac{\phi^{\prime}}{\phi}\right)+C_{1}^{2}\left(\frac{\phi^{\prime}}{\phi}\right)^{2}, \\
u^{3}=C_{1}^{3}\left(\frac{\phi^{\prime}}{\phi}\right)^{3}+3 C_{1}^{2} C_{0}\left(\frac{\phi^{\prime}}{\phi}\right)^{2}+3 C_{1} C_{0}^{2}\left(\frac{\phi^{\prime}}{\phi}\right)+C_{0}^{3} .
\end{gathered}
$$

Now substituting the values of $u, u^{2}, u^{3}, u^{\prime \prime}$ into (38) and then equating the coefficients of $\phi^{0}, \phi^{-1}, \phi^{-2}, \phi^{-3}$ to zero, we, respectively, obtain

$$
\begin{gathered}
-\frac{1}{2} C_{0}^{3}+\frac{3}{2} \omega C_{0}^{2}-\omega^{2} C_{0}=0, \\
C_{1} \phi^{\prime \prime \prime}-\omega^{2} C_{1} \phi^{\prime}-\frac{3}{2} C_{0}^{2} C_{1} \phi^{\prime}+3 \omega C_{0} C_{1} \phi^{\prime}=0, \\
-3 C_{1} \phi^{\prime \prime} \phi^{\prime}-\frac{3}{2} C_{0} C_{1}^{2}\left(\phi^{\prime}\right)^{2}+\frac{3}{2} \omega C_{1}^{2}\left(\phi^{\prime}\right)^{2}=0, \\
2 C_{1}\left(\phi^{\prime}\right)^{3}-\frac{1}{2} C_{1}^{3}\left(\phi^{\prime}\right)^{3}=0 .
\end{gathered}
$$

Solving (41), we get

$$
C_{0}=0, \omega, 2 \omega .
$$

Solving (44), we get

$$
C_{1}= \pm 2, \quad C_{1} \neq 0 .
$$

From (42) and (43), we get

$$
\phi^{\prime}(\xi)=-M E \exp (-L M \xi) .
$$

Integrating (47), we obtain

$$
\phi(\xi)=\frac{E \exp (L M \xi)+L F}{L},
$$

where $L=\left(\omega^{2}+(3 / 2) C_{0}^{2}-3 \omega C_{0}\right), M=2 /\left(\omega-C_{0}\right) C_{1}$, and $E$, $F$ are constants of integration.

Substituting $\xi, \phi(\xi)$ and $\phi^{\prime}(\xi)$ from (47) and (48) into (39), we obtain

$$
u(x, t)=C_{0}-C_{1}\left(\frac{L M E \exp (-L M(x-\omega t))}{E \exp (-L M(x-\omega t))+L F}\right) .
$$

Case 1. When $C_{0}=\omega$, (49) yields trivial solution. So, this case is rejected.

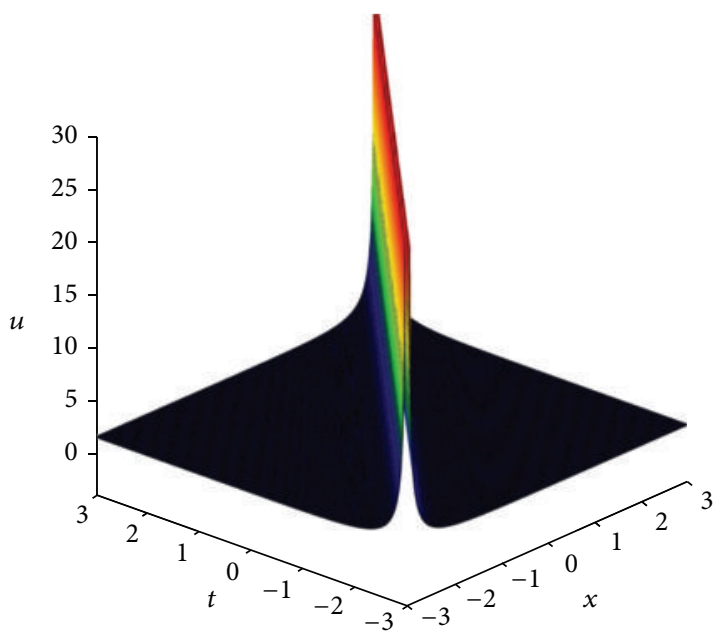

FIGURE 1: Singular soliton profile of (24) with wave speed $\omega=1$, $d=1$, and $-3 \leq x, t \leq 3$.

Case 2. When $C_{0}=0$ and $C_{1}= \pm 2$, executing the parallel course of action described in Section 3.1 (Case 2), putting the values of $L$ and $M(49)$ yields,

$$
\begin{aligned}
& u_{1,2}(x, t)=\omega\left(1 \pm \tanh \left(\frac{\omega}{2}(x-\omega t)\right)\right), \\
& u_{3,4}(x, t)=\omega\left(1 \pm \operatorname{coth}\left(\frac{\omega}{2}(x-\omega t)\right)\right) .
\end{aligned}
$$

Substituting (49) and (50) into (36), we obtain

$$
\begin{gathered}
H_{1}(x, t)=\frac{\omega^{2}}{2} \operatorname{sech}^{2}\left(\frac{\omega}{2}(x-\omega t)\right), \\
H_{2}(x, t)=-\frac{\omega^{2}}{2} \operatorname{cosech}^{2}\left(\frac{\omega}{2}(x-\omega t)\right) .
\end{gathered}
$$

Case 3. When $C_{0}=2 \omega$ and $C_{1}= \pm 2$, we get the same results like (50)-(53).

\section{Physical Explanation}

In this section, we will put forth the physical explanation and the graphical representation of determined traveling wave solutions of nonlinear evolution equations through coupled Konno-Oono equations and the variant Boussinesq equations.

\subsection{Explanations}

(i) The equations (24) and (25) are complex soliton solutions. The shape of (24) is known as singular soliton, and the shape of (25) is known as kink soliton. Figures 1 and 2 represent the modulus shape of (24) and (25) with wave speed $\omega=1, d=1$ and wave speed $\omega=1, d=2$, respectively, within the interval $-3 \leq x, t \leq 3$. The disturbance of (24) and (25) is in the positive $x$-direction for positive values of wave speed $\omega$. If we take negative values of wave speed $\omega$, 


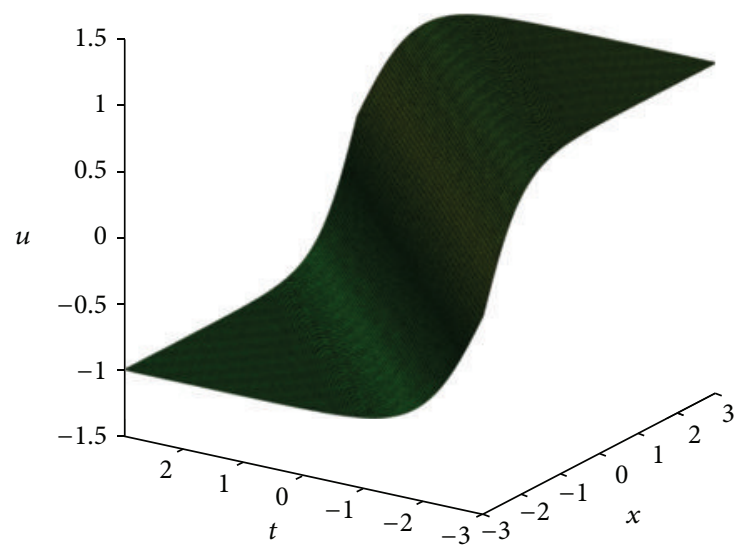

FIGURE 2: Kink wave profile of (25) with wave speed $\omega=1, d=2$, and $-3 \leq x, t \leq 3$.

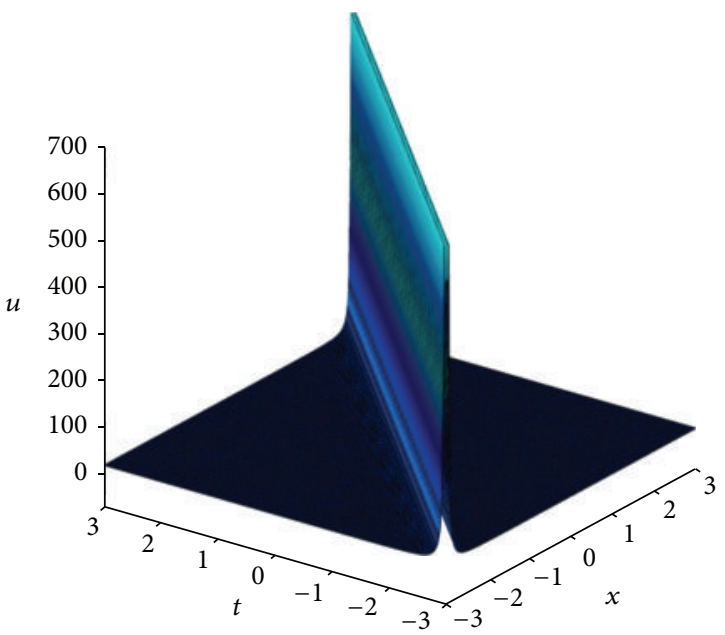

Figure 3: Soliton wave of (26) with wave speed $\omega=-2, d=4$, and $-3 \leq x, t \leq 3$.

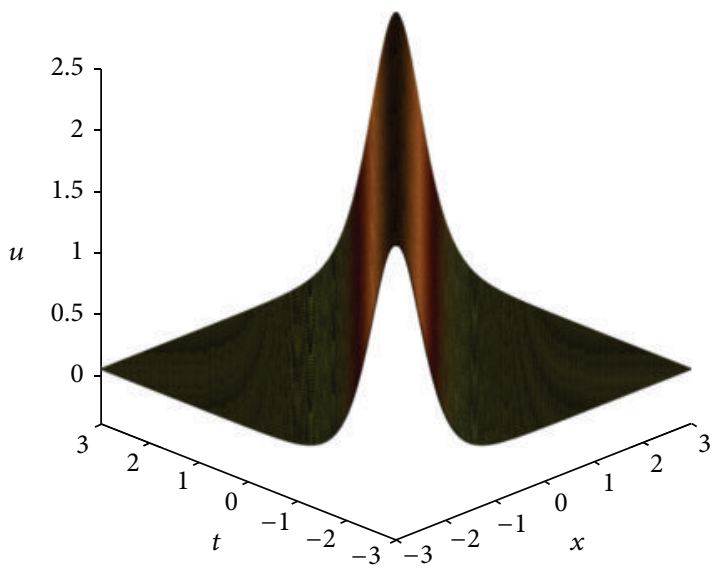

FIGURE 4: Bell-shaped wave profile of (27) with wave speed $\omega=1$, $d=2$, and $-3 \leq x, t \leq 3$.

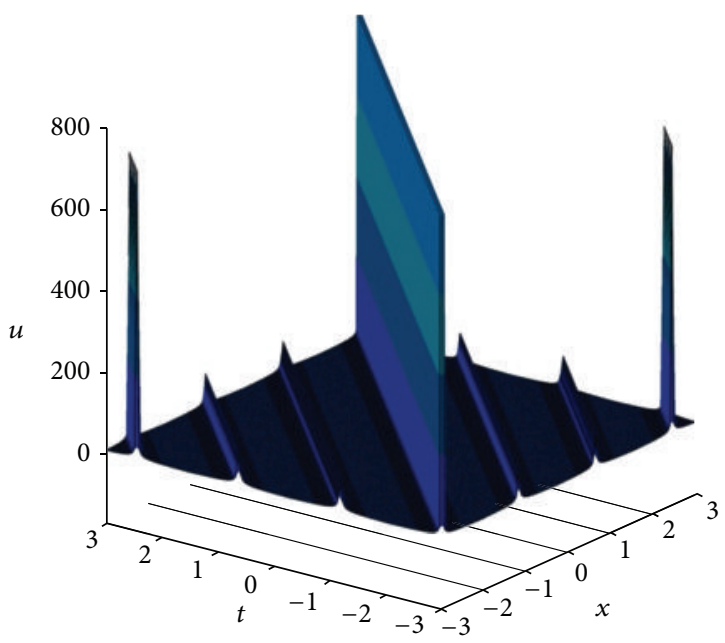

FIGURE 5: Modulus plot of periodic wave, shape of (28) with wave speed $\omega=1, d=-3$, and $-3 \leq x, t \leq 3$.

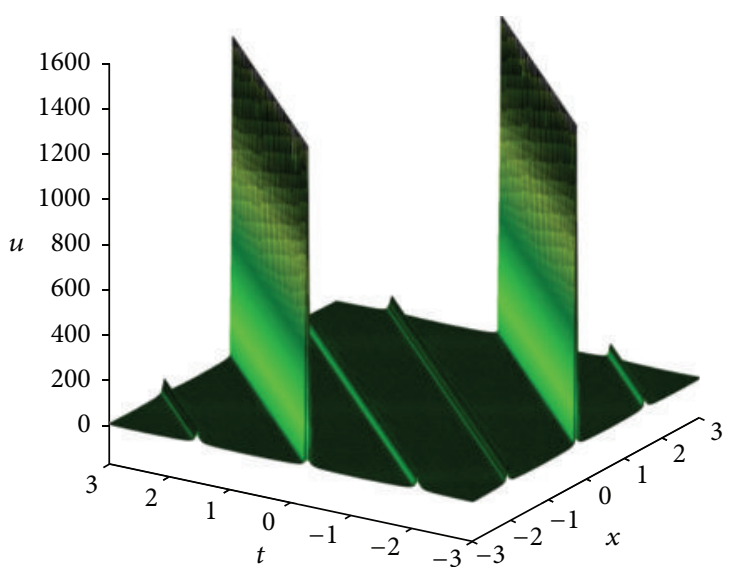

FIGURE 6: Modulus plot of periodic wave, profile of (29) with wave speed $\omega=1, d=-3$, and $-3 \leq x, t \leq 3$.

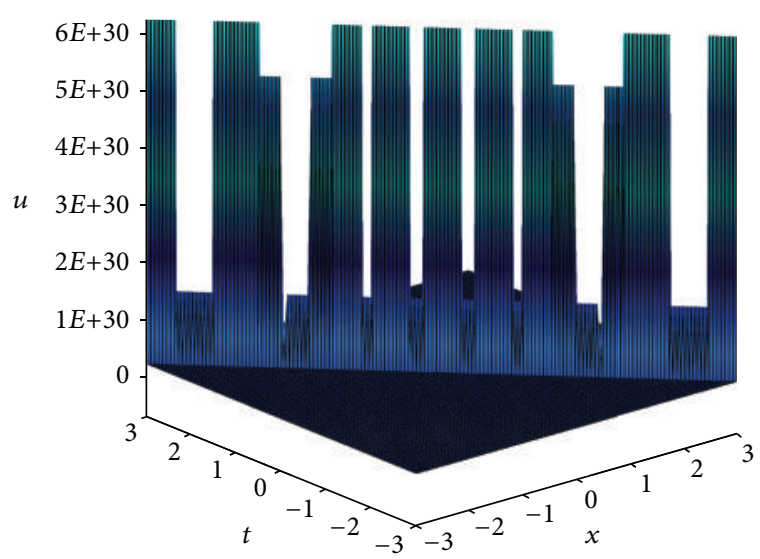

FIgURE 7: $3 \mathrm{~d}$ plot of periodic wave solution, shape of (30) with wave speed $\omega=-1, d=-4$, and $-3 \leq x, t \leq 3$. 


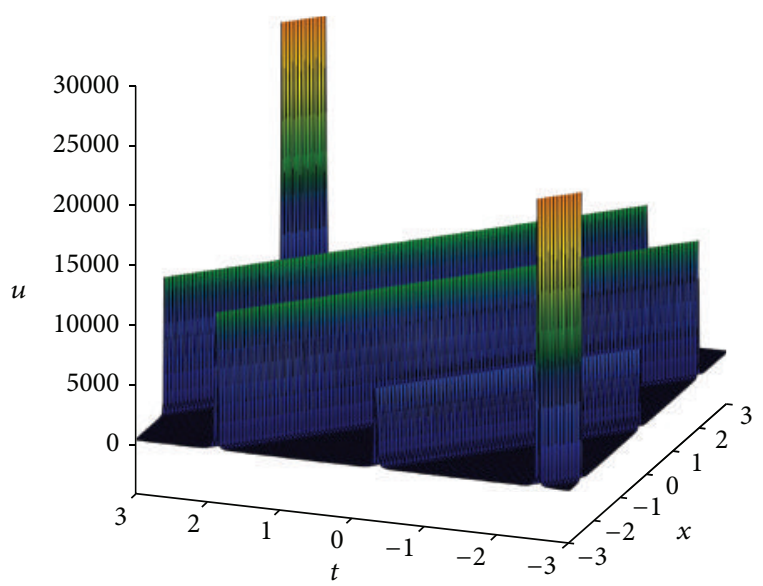

FIGURE 8: 3d plot of periodic wave solution, profile of (31) with wave speed $\omega=-1, d=-2$, and $-3 \leq x, t \leq 3$.

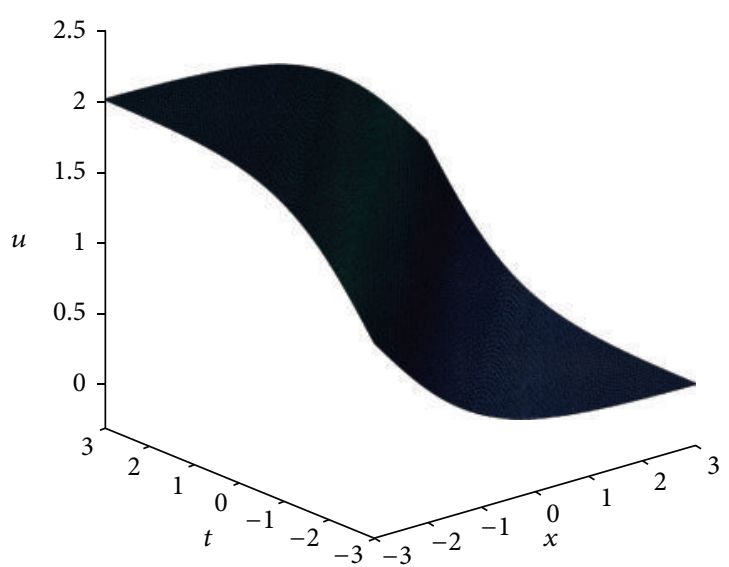

FIGURE 9: Kink wave, profile of (50) with wave speed $\omega=1$ and $-3 \leq x, t \leq 3$.

then the disturbance of (24) and (25) will be in the negative $x$-direction.

(ii) Equations (26) and (27) are soliton solutions. Figure 3 shows the shape of singular soliton of (26) with wave speed $\omega=-2, d=4$, and $-3 \leq x, t \leq 3$, and Figure 4 shows bell-shaped soliton of (27) with wave speed $\omega=1, d=2$, and $-3 \leq x, t \leq 3$. The propagation or disturbance of (26), represented in Figure 3, is in the negative $x$-direction. And the propagation or disturbance of (27), represented in Figure 4, is in the positive $x$-direction.

(iii) Figures 5, 6, 7, and 8 corresponding to the shape of (28)-(31) are traveling wave solutions, which are periodic.

(iv) Figure 9 represents the profile of (50) that is kink solution with wave speed $\omega=1$ and $-3 \leq x, t \leq 3$.

(v) Figure 10 represents the silhouette of (51) that is singular kink solution with wave speed $\omega=9$ and $-3 \leq x, t \leq 3$.

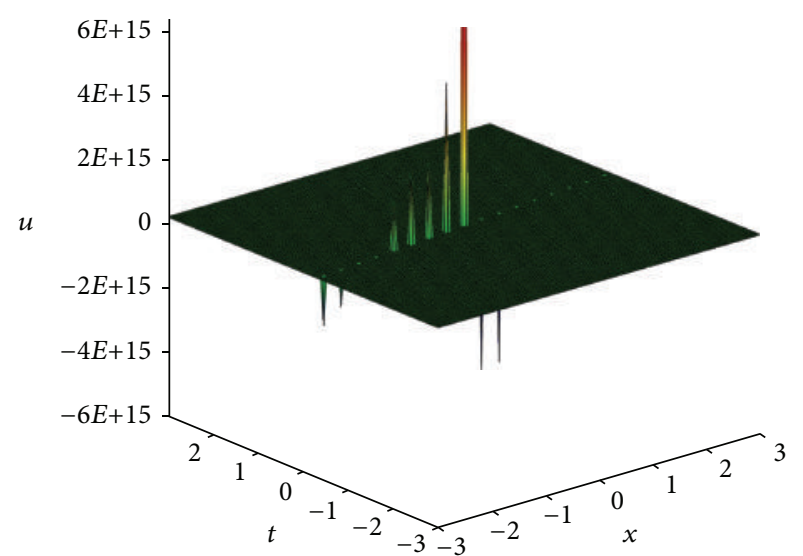

FIGURE 10: Singular kink soliton, shape of (51) with wave speed $\omega=9$ and $-3 \leq x, t \leq 3$.

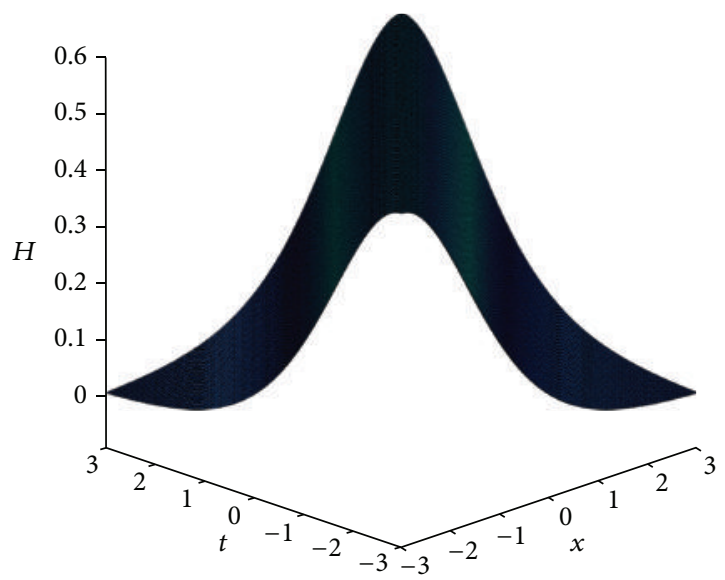

FIGURE 11: Bell-shaped soliton, profile of (52) with wave speed $\omega=1$ and $-3 \leq x, t \leq 3$.

(vi) Figure 11 represents the shadow of (52) that is bellshaped solution with wave speed $\omega=1$ and $-3 \leq x$, $t \leq 3$.

(vii) Figure 12 represents the profile of (53) that is soliton solution with wave speed $\omega=1$ and $-3 \leq x, t \leq 3$.

The disturbances represented in Figures 9-12 are in the positive $x$-direction.

4.2. Graphical Representation. Some of our obtained traveling wave solutions are represented in the following figures with the aid of commercial software Maple.

\section{Conclusions}

In this paper, the MSE method has been employed for analytic treatment of two nonlinear coupled partial differential equations. The MSE method requires wave transformation formulae. Via MSE method traveling wave solutions, kink solutions, bell-shaped solutions of coupled Konno-Oono equations, and the variant Boussinesq equations were derived. The 


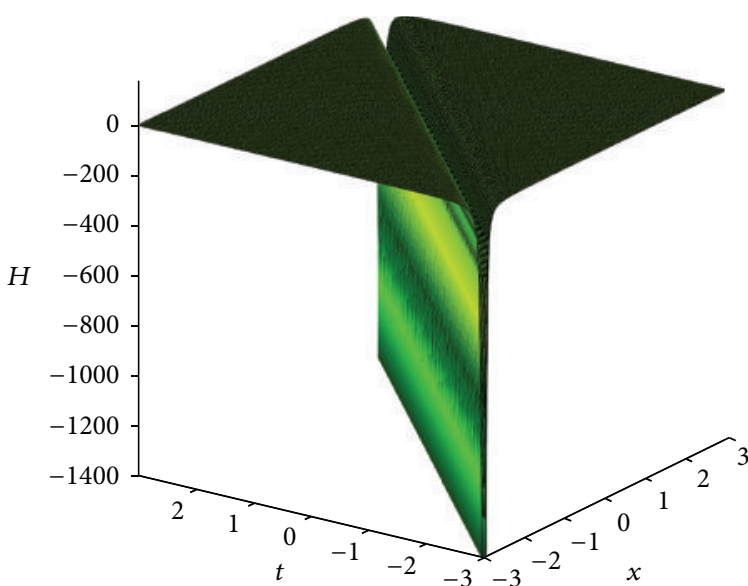

FIGURE 12: Singular soliton, shape of (53) with wave speed $\omega=1$ and $-3 \leq x, t \leq 3$.

procedure is simple, direct, and constructive. Without the help of a computer algebra system all examples in this paper show the efficiency of MSE method.

\section{References}

[1] A. J. M. Jawad, M. D. Petković, and A. Biswas, "Modified simple equation method for nonlinear evolution equations," Applied Mathematics and Computation, vol. 217, no. 2, pp. 869-877, 2010.

[2] K. Khan and M. A. Akbar, "Exact and solitary wave solutions for the Tzitzeica-Dodd-Bullough and the modified KdV-ZakharovKuznetsov equations using the modified simple equation method," Ain Shams Engineering Journal. In press.

[3] K. Khan, M. A. Akbar, and N. H. M. Ali, "The modified simple equation method for exact and solitary wave solutions of nonlinear evolution equation: the GZK-BBM equation and right-handed noncommutative burgers equations," ISRN Mathematical Physics, vol. 2013, Article ID 146704, 5 pages, 2013.

[4] E. M. E. Zayed and S. A. H. Ibrahim, "Exact solutions of nonlinear evolution equations in mathematical physics using the modified simple equation method," Chinese Physics Letters, vol. 29, no. 6, Article ID 060201, 2012.

[5] E. G. Fan, "Extended tanh-function method and its applications to nonlinear equations," Physics Letters A, vol. 277, no. 4-5, pp. 212-218, 2000.

[6] M. A. Abdou, "The extended tanh method and its applications for solving nonlinear physical models," Applied Mathematics and Computation, vol. 190, no. 1, pp. 988-996, 2007.

[7] J.-H. He and X.-H. Wu, "Exp-function method for nonlinear wave equations," Chaos, Solitons \& Fractals, vol. 30, no. 3, pp. 700-708, 2006.

[8] M. A. Akbar and N. H. M. Ali, "Exp-function method for Duffing Equation and new solutions of $(2+1)$ dimensional dispersive long wave equations," Program in Applied Mathematics, vol. 1, no. 2, pp. 30-42, 2011.

[9] H. Naher, A. F. Abdullah, and M. A. Akbar, "The Exp-function method for new exact solutions of the nonlinear partial differential equations," International Journal of Physical Sciences, vol. 6, no. 29, pp. 6706-6716, 2011.

[10] H. Naher, F. A. Abdullah, and M. Ali Akbar, "New traveling wave solutions of the higher dimensional nonlinear partial differential equation by the exp-function method," Journal of Applied Mathematics, vol. 2012, Article ID 575387, 14 pages, 2012.

[11] A. Bekir and A. Boz, "Exact solutions for nonlinear evolution equations using Exp-function method," Physics Letters A, vol. 372, no. 10, pp. 1619-1625, 2008.

[12] G. Adomian, Solving Frontier Problems of Physics: The Decomposition Method, Kluwer Academic, Dodrecht, The Netherlands, 1994.

[13] Y. Zhou, M. Wang, and Y. Wang, "Periodic wave solutions to a coupled KdV equations with variable coefficients," Physics Letters A, vol. 308, no. 1, pp. 31-36, 2003.

[14] Sirendaoreji, "New exact travelling wave solutions for the Kawahara and modified Kawahara equations," Chaos, Solitons and Fractals, vol. 19, no. 1, pp. 147-150, 2004.

[15] A. T. Ali, "New generalized Jacobi elliptic function rational expansion method," Journal of Computational and Applied Mathematics, vol. 235, no. 14, pp. 4117-4127, 2011.

[16] Y. He, S. Li, and Y. Long, "Exact solutions of the Klein-Gordon equation by modified exp-function method," International Mathematical Forum, vol. 7, no. 1-4, pp. 175-182, 2012.

[17] M. A. Akbar, N. H. M. Ali, and E. M. E. Zayed, "Abundant exact traveling wave solutions of the generalized Bretherton equation via $\left(G^{\prime} / G\right)$-expansion method," ommunications in Theoretical Physics, vol. 57, no. 2, pp. 173-178, 2012.

[18] M. A. Akbar, N. H. M. Ali, and E. M. E. Zayed, "A generalized and improved $\left(G^{\prime} / G\right.$-expansion method for nonlinear evolution equations," Mathematical Problems in Engineering, vol. 2012, Article ID 459879, 22 pages, 2012.

[19] M. A. Akbar, N. H. M. Ali, and S. T. Mohyud-Din, "The alternative $\left(G^{\prime} / G\right)$-expansion method with generalized Riccati equation: application to fifth order (1+1)-dimensional CaudreyDodd-Gibbon equation," International Journal of Physical Sciences, vol. 7, no. 5, pp. 743-752, 2012.

[20] M. A. Akbar, N. H. M. Ali, and S. T. Mohyud-Din, "Some new exact traveling wave solutions to the $(3+1)$-dimensional Kadomtsev-Petviashvili equation," World Applied Sciences Journal, vol. 16, no. 11, pp. 1551-1558, 2012.

[21] E. M. E. Zayed and S. Al-Joudi, "Applications of an extended $\left(G^{\prime} / G\right)$-expansion method to find exact solutions of nonlinear PDEs in mathematical physics," Mathematical Problems in Engineering, vol. 2010, Article ID 768573, 19 pages, 2010.

[22] E. M. E. Zayed, "Traveling wave solutions for higher dimensional nonlinear evolution equations using the $\left(G^{\prime} / G\right)$ expansion method," Journal of Applied Mathematics \& Informatics, vol. 28, pp. 383-395, 2010.

[23] E. M. E. Zayed and K. A. Gepreel, “The $\left(G^{\prime} / G\right)$-expansion method for finding traveling wave solutions of nonlinear partial differential equations in mathematical physics," Journal of Mathematical Physics, vol. 50, no. 1, pp. 013502-013514, 2009.

[24] M. Wang, X. Li, and J. Zhang, “The $\left(G^{\prime} / G\right)$-expansion method and travelling wave solutions of nonlinear evolution equations in mathematical physics," Physics Letters A, vol. 372, no. 4, pp. 417-423, 2008.

[25] M. A. Akbar and N. H. M. Ali, "The alternative $\left(G^{\prime} / G\right)$ expansion method and its applications to nonlinear partial differential equations," International Journal of Physical Sciences, vol. 6, no. 35, pp. 7910-7920, 2011.

[26] A. R. Shehata, "The traveling wave solutions of the perturbed nonlinear Schrödinger equation and the cubic-quintic 
Ginzburg Landau equation using the modified $\left(G^{\prime} / G\right)$ expansion method," Applied Mathematics and Computation, vol. 217, no. 1, pp. 1-10, 2010.

[27] L.-M. Shi, L.-F. Zhang, H. Meng, H.-W. Zhao, and S.-P. Zhou, "A method to construct Weierstrass elliptic function solution for nonlinear equations," International Journal of Modern Physics B, vol. 25, no. 14, pp. 1931-1939, 2011.

[28] S. T. Mohyud-Din and M. A. Noor, "Homotopy perturbation method for solving fourth-order boundary value problems," Mathematical Problems in Engineering, vol. 2007, Article ID 98602, 15 pages, 2007.

[29] S. T. Mohyud-Din and M. A. Noor, "Homotopy perturbation method for solving partial differential equations," Zeitschrift fur Naturforschung A, vol. 64, no. 3-4, pp. 157-170, 2009.

[30] S. T. Mohyud-Din, A. Yıldırım, and S. A. Sezer, "Numerical soliton solutions of improved Boussinesq equation," International Journal of Numerical Methods for Heat \& Fluid Flow, vol. 21, no. 6-7, pp. 822-827, 2011.

[31] M. L. Wang, "Solitary wave solutions for variant Boussinesq equations," Physics Letters A, vol. 199, no. 3-4, pp. 169-172, 1995.

[32] E. M. E. Zayed, H. A. Zedan, and K. A. Gepreel, "On the solitary wave solutions for nonlinear Hirota-Satsuma coupled KdV of equations," Chaos, Solitons and Fractals, vol. 22, no. 2, pp. 285303, 2004.

[33] R. Hirota, "Exact envelope-soliton solutions of a nonlinear wave equation," Journal of Mathematical Physics, vol. 14, pp. 805-809, 1973.

[34] R. Hirota and J. Satsuma, "Soliton solutions of a coupled Korteweg-de Vries equation,” Physics Letters A, vol. 85, no. 8-9, pp. 404-408, 1981.

[35] W. Malfliet, "Solitary wave solutions of nonlinear wave equations," American Journal of Physics, vol. 60, no. 7, pp. 650-654, 1992.

[36] N. H. Abdel-All, M. A.-A. Abdel-Razek, and A.-A. K. Seddeek, "Expanding the tanh-function method for solving nonlinear equations," Applied Mathematics, vol. 2, no. 9, pp. 1096-1104, 2011.

[37] K. Konno and H. Oono, "New coupled integrable dispersionless equations," Journal of the Physical Society of Japan, vol. 63, pp. 377-378, 1994. 


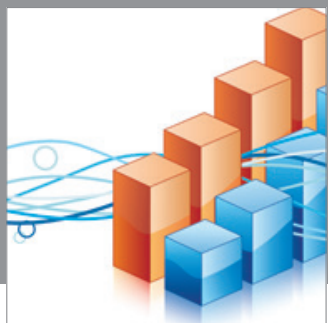

Advances in

Operations Research

mansans

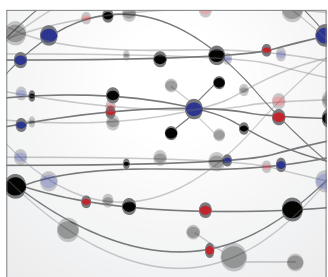

The Scientific World Journal
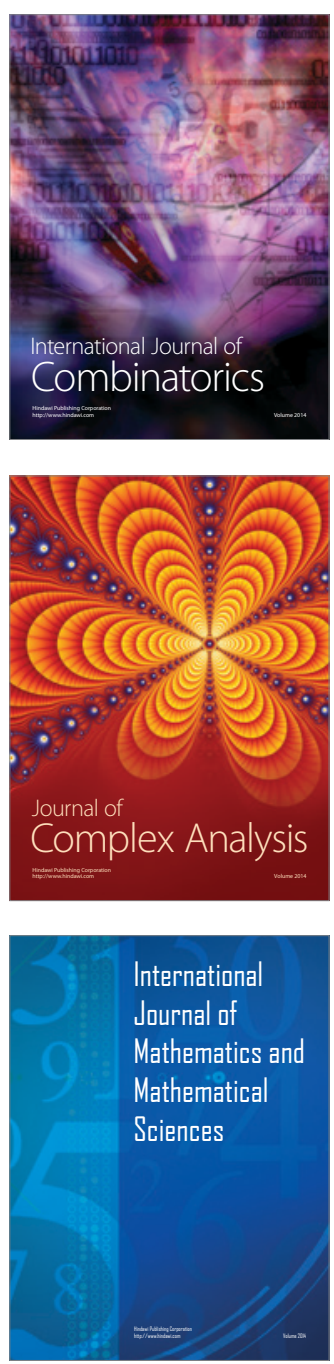
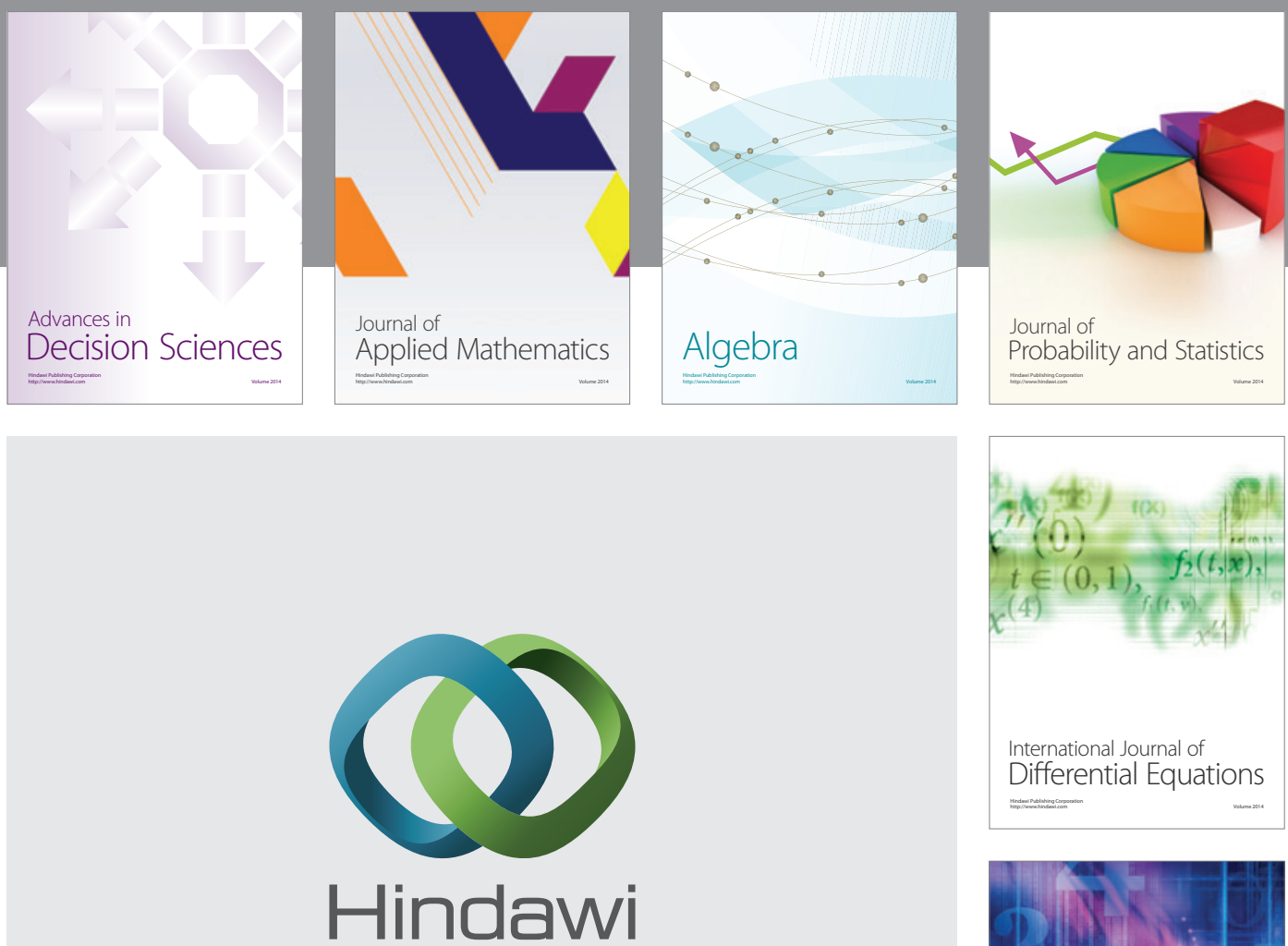

Submit your manuscripts at http://www.hindawi.com
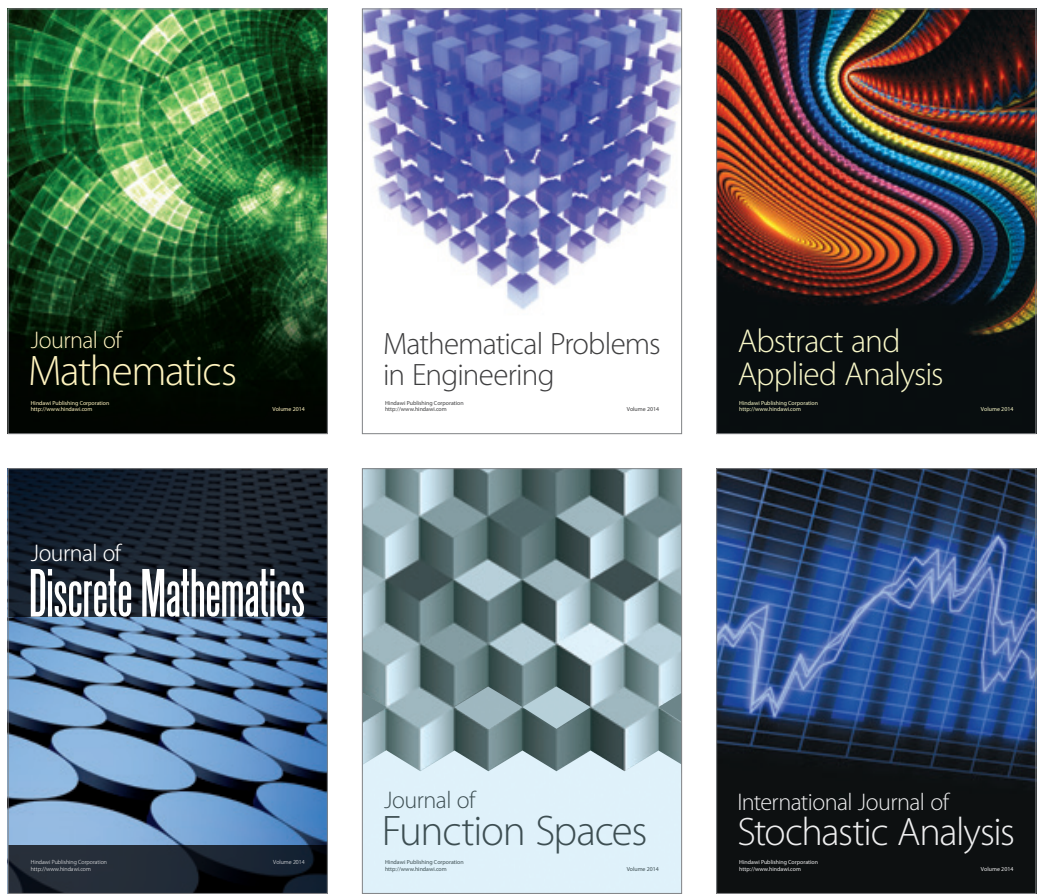

Journal of

Function Spaces

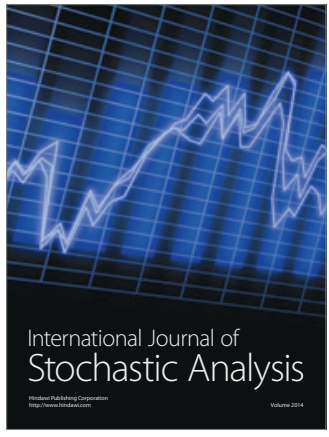

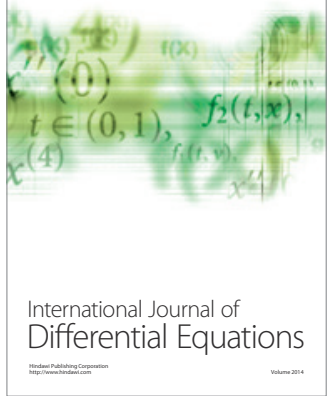
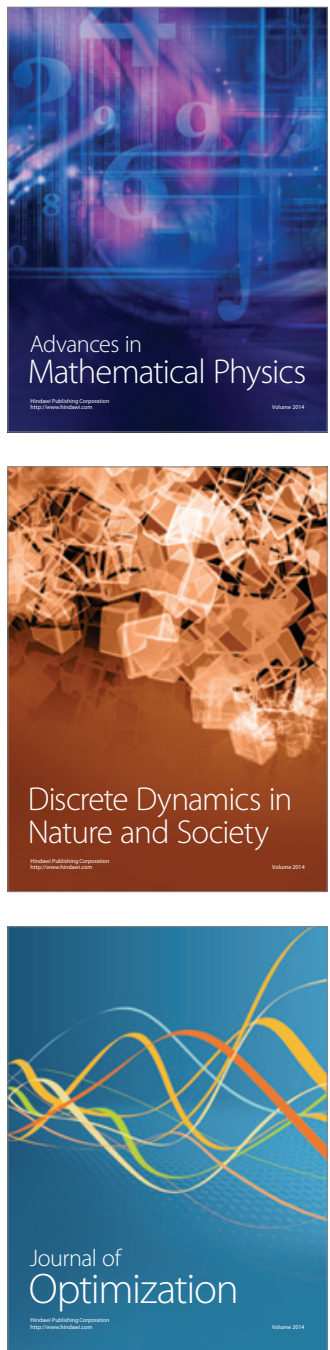